\title{
Vaccine Development, AIDS
}

National Cancer Institute

\section{Source}

National Cancer Institute. Vaccine Development, AIDS. NCI Thesaurus. Code C15866.

Vaccines or candidate vaccines containing inactivated HIV or some of its component antigens and designed to prevent AIDS. Some vaccines containing antigens are recombinantly produced. 